\title{
Overproducing the BAM complex improves secretion of difficult-to-secrete recombinant autotransporter chimeras
}

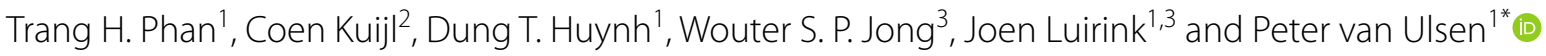

\begin{abstract}
Monomeric autotransporters have been used extensively to transport recombinant proteins or protein domains to the cell surface of Gram-negative bacteria amongst others for antigen display. Genetic fusion of such antigens into autotransporters has yielded chimeras that can be used for vaccination purposes. However, not every fusion construct is transported efficiently across the cell envelope. Problems occur in particular when the fused antigen attains a relatively complex structure in the periplasm, prior to its translocation across the outer membrane. The latter step requires the interaction with periplasmic chaperones and the BAM ( $\beta$-barrel assembly machinery) complex in the outer membrane. This complex catalyzes insertion and folding of $\beta$-barrel outer membrane proteins, including the $\beta$-barrel domain of autotransporters. Here, we investigated whether the availability of periplasmic chaperones or the BAM complex is a limiting factor for the surface localization of difficult-to-secrete chimeric autotransporter constructs. Indeed, we found that overproduction of in particular the BAM complex, increases surface display of difficult-tosecrete chimeras. Importantly, this beneficial effect appeared to be generic not only for a number of monomeric autotransporter fusions but also for fusions to trimeric autotransporters. Therefore, overproduction of BAM might be an attractive strategy to improve the production of recombinant autotransporter constructs.
\end{abstract}

Keywords: Type V secretion systems, Hbp, Surface display, BAM complex, Outer membrane proteins

\section{Introduction}

For many biotechnology applications, including recombinant protein production or vaccine development, it is considered an advantage to secrete proteins into the extra-cellular milieu or to display them on the surface of the producing bacteria [18, 19, 36, 54]. Gram-negative bacteria have a complex cell envelope that consists of a cytoplasmic membrane and an outer membrane (OM) enclosing the periplasmic space with the peptidoglycan layer. Multiple secretion pathways have evolved to transport proteins across the two membranes [3]. Most

\footnotetext{
*Correspondence: j.p.van.ulsen@vu.nl

${ }^{1}$ Department of Molecular Microbiology, Amsterdam Institute

of Molecular and Life Sciences, Vrije Universiteit Amsterdam, Amsterdam, The Netherlands

Full list of author information is available at the end of the article
}

pathways require multi-protein secretion complexes that span the cell envelope. By contrast, the five subclasses of the type $\mathrm{V}$ (or autotransporter) secretion pathway (type $\mathrm{Va}-\mathrm{Ve}$ ) are fairly simple and encoded by one or two genes [8]. The secreted proteins of these systems cross the cell envelope in two consecutive steps. The type $\mathrm{V}$ systems, in general, comprise only a single gene encoding a signal peptide at the $\mathrm{N}$-terminus, a secreted protein domain often called the passenger and a $\beta$-barrel domain involved in transport of the passenger across the outer membrane $[8,30,54]$. An exception is formed by the Two Partner secretion systems, that are classified as type $\mathrm{Vb}$ and consist of two genes encoding the secreted protein and a dedicated transporter. Furthermore, while the passengers of the type $\mathrm{Vc}-\mathrm{Ve}$ subclasses remain attached to their $\beta$-barrel domains, most passengers of original author(s) and the source, provide a link to the Creative Commons licence, and indicate if changes were made. The images or other third party material in this article are included in the article's Creative Commons licence, unless indicated otherwise in a credit line to the material. If material is not included in the article's Creative Commons licence and your intended use is not permitted by statutory regulation or exceeds the permitted use, you will need to obtain permission directly from the copyright holder. To view a copy of this licence, visit http://creativecommons.org/licenses/by/4.0/. The Creative Commons Public Domain Dedication waiver (http://creativeco mmons.org/publicdomain/zero/1.0/) applies to the data made available in this article, unless otherwise stated in a credit line to the data. 
the type Va subclass, the classical autotransporters, are proteolytically cleaved from their $\beta$-barrel domain after which they are released into the extra-cellular milieu or remain bound to the cell surface via non-covalent interactions (see Fig. 1 for the gene organization in classical autotransporters). Despite their apparent simplicity, type $\mathrm{V}$ secretion systems secrete some of the largest proteins encoded by bacterial genomes. They function in most cases as adhesin, protease, or toxin to support bacterial virulence [30].

The signal peptide is required for targeting to and transfer across the Sec-translocon in the cytoplasmic membrane. Upon translocation into the periplasm, the signal peptide is cleaved and the mature autotransporter engages periplasmic chaperones such as SurA to maintain translocation competence and trigger targeting to the BAM complex, which functions as a generic foldase and insertase for $\beta$-barrel outer membrane proteins (OMPs) $[9,22,32]$. The interaction of the BAM complex with the $\beta$-barrel domain of autotransporters not only results in their insertion into the $\mathrm{OM}$. it also facilitates the transport of the passenger to the cell surface $[8,30$, 54]. Recent structural studies show that the central component of the BAM complex, the integral OMP BamA, interacts with nascent OMPs to aid $\beta$-barrel folding [27, 49]. Similarly, contacts were found between BamA and the $\beta$-domain and passenger of the classical autotransporter EspP while being translocated across the OM, indicating a intricate contact between BamA and the nascent autotransporter during this process [6]. In addition to BamA, the BAM complex of Escherichia coli and other gammaproteobacteria consists of four accessory lipoproteins (BamB-E), although variations in composition have been found in other bacterial classes [56].

The classical autotransporters are widely applied for secretion and cell-surface display of heterologous proteins, e.g. enzymes for biotechnical applications or antigens for vaccine development $[19,50,54]$. We have studied the structure and secretion mechanism of the
Escherichia coli autotransporter hemoglobin protease (Hbp), which helped to develop a vaccine platform in which multiple antigens are displayed at the surface of non-pathogenic bacteria and outer membrane vesicles (OMVs) [5, 13, 18, 24, 42]. Sequences encoding the antigen of choice were inserted into $h b p$, replacing those encoding subdomains of the $\mathrm{Hbp}$ passenger that extend from the $\beta$-helical stem that this passenger forms $[13,18,33]$. To expose such antigens on the surface of the bacterial cell or OMVs, Hbp-Display constructs $(\mathrm{HbpD})$ were made that lack the autocatalytic cleavage site thus preventing release of the passenger into the extracellular milieu [18]. However, the translocation capacity of $\mathrm{HbpD}$ appeared to be limited by the number, size and in particular the structural complexity of the fused cargo antigens $[13,16,41]$.

Previously, we have attempted to expand the secretion capacity of the Hbp system by testing adaptations to the $\beta$-barrel domain and by replacing it for the $\beta$-barrel domain of another autotransporter [14, 39] but with limited success. Here we examined whether increased expression of generic components of the secretion route of autotransporters can improve secretion of overproduced and difficult-to-secrete (DTS) recombinant $\mathrm{Hbp}$ chimeras. During secretion $\mathrm{Hbp}$ has been shown to interact with the periplasmic chaperone SurA and the BamA and BamB components of the BAM complex [40], whereas Hbp constructs that are blocked in secretion are degraded by DegP, a periplasmic protease upregulated upon cell-envelope stress $[16,47]$. In addition, the periplasmic chaperones SurA and Skp and BAM complex subunits BamA, BamB and BamD with $E$. coli autotransporter EspP have been reported $[11,12]$. Here, we investigated the possibility that the limited availability of these factors is a bottleneck in the secretion of DTS HbpD chimeras. Indeed, we found that overproduction of the BAM complex markedly improves surface display of such $\mathrm{Hbp}$ chimeras. Coexpression of various chaperones also had a positive albeit less general influence on display efficiency.

\footnotetext{
(See figure on next page.)

Fig. 1 Co-overexpression of the BAM complex improved expression of HbpD-ELL. A Schematic representation of HbpD, HbpD-ESAT6 and HbpD-ELL with models of the folded passengers indicating the disulfide-bonded L9L9 hairpin blocking secretion. Indicated are further the mutated cleavage site in between the passenger and $\beta$-barrel domain (red), the $\mathrm{N}$-terminal signal peptide (dotted box), the passenger domain (blue), and the C-terminal $\beta$-domain (pale green). Boxes d1 to d5 (light blue) represent regions of $\mathrm{Hbp}$ that can be exchanged for heterologous protein segments [18]. In HbpD-ESAT6, mycobacterial antigen ESAT6 (orange) replaces d1, in HbpD-ELL the L9L9 hairpin replaces d4 (green). B, C Coomassie-stained SDS-PAGE gels of whole cell lysates from E. coli BL21 (DE3) cultures expressing HbpD-ESAT6 (B) or HbpD-ELL (C), both with or without induction for extra BAM complex. The final concentration of inducers IPTG (BAM) and Rhamnose (HbpD) are indicated above the gel-images. The protein bands representing BamA, BamC, OmpF/C, OmpA, HbpD-ESAT6 and HbpD-ELL are indicated by "<". D Western blots incubated with antisera recognizing ESAT6, BamA, DegP and SurA
} 
A

$\mathrm{HbpD}$

1

\begin{tabular}{l|l|l|l|}
2 & 3 & 4 & 5
\end{tabular}

HbpD-ESAT6

:-

\begin{tabular}{ll|l|l|l}
2 & 3 & 4 & 5
\end{tabular}

5

HbpD-ELL

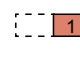

B

HbpD-ESAT6

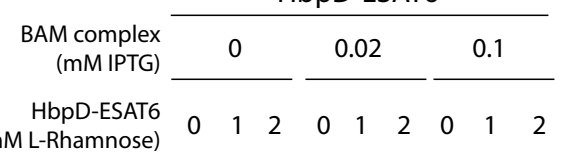

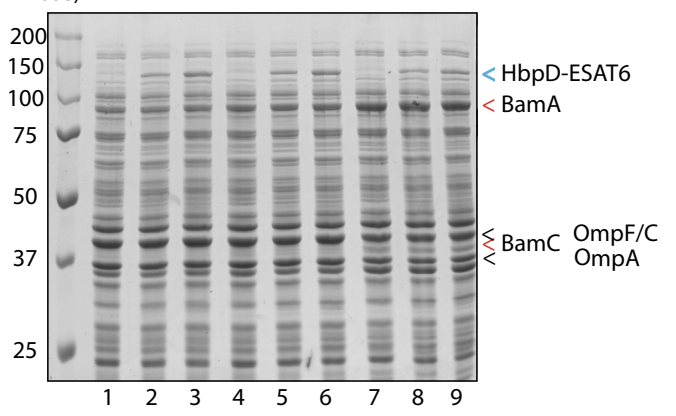

C

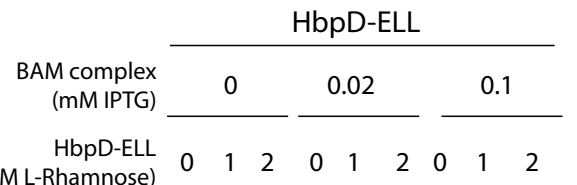

\begin{tabular}{lllllllllll} 
(mM L-Rhamnose) & 0 & 1 & 2 & 0 & 1 & 2 & 0 & 1 & 2 \\
\hline
\end{tabular}

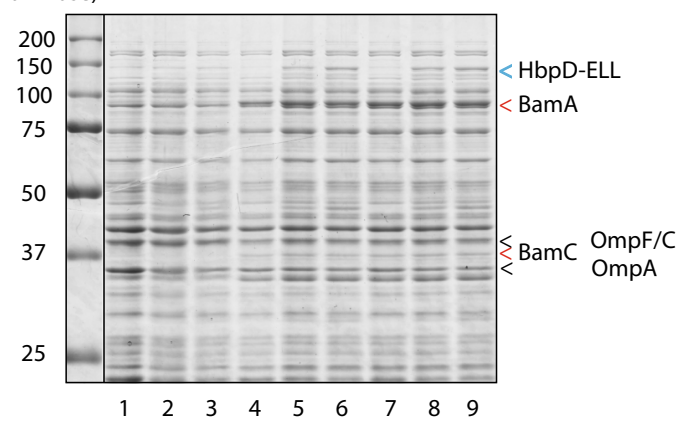

D

HbpD-ESAT6

HbpD-ELL

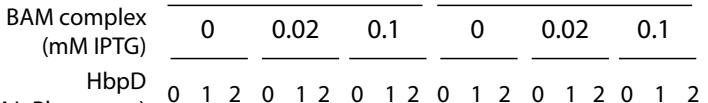

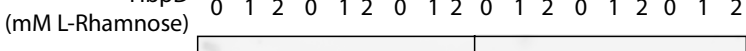

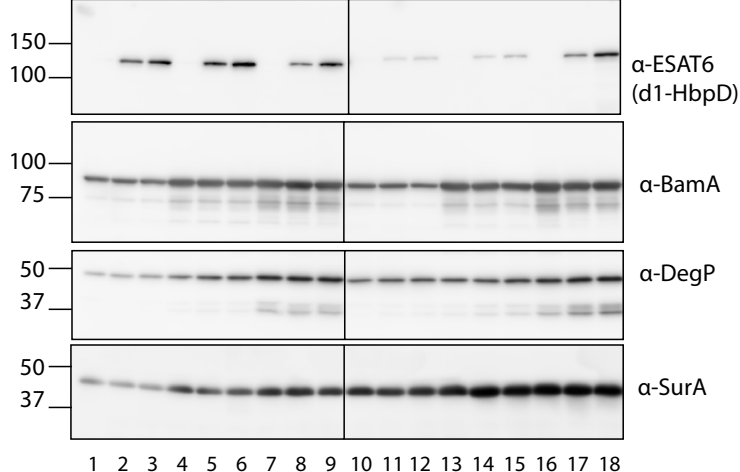

Fig. 1 (See legend on previous page.) 


\section{Results}

\section{Co-overexpression of the BAM complex improved} expression of HbpD-ELL

In earlier studies, we have reported on the limited tolerance of $\mathrm{Hbp}$ to transport folded heterologous protein domains across the outer membrane where such DTS constructs showed a decreased secretion efficiency when compared to wild-type Hbp or efficiently-secreted derivatives [13, 16, 41]. Examples of DTS inserts are the calmodulin domain that forms a stable fold in the presence of calcium ions [16] and a single-chain antibody domain that includes two disulfide bonds [14]. To systematically investigate the limits of $\mathrm{Hbp}$ secretion, we previously constructed a DTS Hbp with an $\alpha$-helical hairpin formed by two stable $\alpha$-helices derived from ribosomal protein L9 of Bacillus stearothermophilus constricted into an hairpin through a disulfide bond formed between cysteine residues engineered at positions 707 and 712 of the Hbp passenger (Fig. 1A, Table 1) [41]. This model passenger was shown to be stalled during translocation across the outer membrane unless the formation of the disulfide bond was prevented [41]. This indicated that single $\alpha$-helices, but not helical hairpins, are compatible with secretion. Introduction of the ESAT6 antigen from Mycobacterium tuberculosis at the $\mathrm{N}$-terminus of the Hbp passenger, where it replaces the protease subdomain of wild-type $\mathrm{Hbp}$ (Fig. 1, region 1), enabled detection of the stalled construct using anti-ESAT6 monoclonal antibodies. Here we have used an uncleaved display variant of this stalled Hbp construct called HbpD-ELL (Fig. 1A). As a control served HbpD-ESAT6 (Fig. 1A), which lacks the helical hairpin and is efficiently secreted to the cell surface [18].

Since the BAM complex was shown to be directly involved in the translocation of $\mathrm{Hbp}$ across the outer membrane [40], we considered the possibility that the endogenous level of BAM-complexes is a limiting factor for efficient secretion of DTS HbpD constructs. To relieve this potential bottleneck, we co-overexpressed the bam operon with either HbpD-ELL or efficiently-secreted HbpD-ESAT6 in E. coli BL21 (DE3). Plasmid pJH114 carries the five genes (bamA-bamE) encoding the BAM complex under control of a single IPTG-inducible trc promoter [37]. To have separately inducible $h b p$ genes, we cloned $h b p D-E L L$ and $h b p D$-ESAT6 downstream of a rhamnose-inducible promoter (Prha) in the pLEMO plasmid [55], which is compatible with pJH114 (Table 2). Expression of the HbpD fusions with and without overexpression of the BAM complex was then compared. To ensure the availability of the additional copies of BAM for $\mathrm{HbpD}$ secretion, expression of the bam operon was induced one hour prior to induction of $\mathrm{HbpD}$ expression for two hours. Samples were taken and analyzed by SDSPAGE followed by staining with Coomassie Brilliant Blue or Western blotting (Fig. 1).

Expression of HbpD-ELL in the absence of IPTGinduced bam expression resulted in a clear growth defect (Additional file 1: Fig. S1) and poor production level when compared to HbpD-ESAT6 (Fig. 1, compare panels $\mathrm{B}$ and $\mathrm{C}$ ), in line with earlier observations for DTS Hbp constructs $[13,41,48]$. Cultures to which no IPTG was added (so with endogenous, or near-endogenous BAM levels) expressed HbpD-ESAT6 $(\sim 125 \mathrm{kDa})$ upon addition of rhamnose at levels detectable both on Coomassie-stained gels and on Western blots incubated with monoclonal antiserum against ESAT6 (Fig. 1B, D), while HbpD-ELL ( $130 \mathrm{kDa})$ was hardly detectable, likely due to stalled secretion and subsequent degradation by the periplasmic protease DegP (Fig. 1C, D [41]). Expression of HbpD-ESAT6 and HbpD-ELL by themselves induced only a moderate increase in DegP levels $[16,48]$ as confirmed by Western blotting (Fig. 1D). IPTG-induced production of BAM from $\mathrm{pJH} 114$ resulted in a dosedependent accumulation of BamA (Fig. 1B-D) indicative of increased BAM complex production. Overexpression of BAM also clearly affected the bacterial growth rate (Additional file 1: Fig. S1) and led to a moderately increased expression of DegP (Fig. 1D, lanes 4, 7, 13,

Table 1 Autotransporter Display constructs used in this study

\begin{tabular}{llll}
\hline Name & Detection antigen & Secretion blocking structure & Cell surface labelling \\
\hline HbpD-ELL & ESAT6 & S-S bonded L9L9 hairpin \\
HbpD-ESAT6 & ESAT6 & - & a-ESAT6 \\
HbpD-SpT2-LL & - & S-S bonded L9L9 hairpin \\
HbpD-SpT2 & - & - & a-ESAT6 \\
HbpD-SpT2-Calm & HA tag & Calmodulin/Ca ${ }^{2+}$ \\
HbpD-SpT2-GFPnb & HA tag & S-S bonded GFP nanobody \\
UpaG-SpT2-Calm & HA tag & Calmodulin/Ca2+ \\
UpaG-GFPnb & HA tag & S-S bonded GFP nanobody \\
\hline
\end{tabular}


Table 2 Plasmids used in this study

\begin{tabular}{|c|c|c|c|c|c|}
\hline Name & ori & Inducible promoter & Inducer & Selection marker & References \\
\hline $\mathrm{pJH1} 14$ & pMB1 & Ptrc & IPTG & bla & [37] \\
\hline pLEMO-HbpD-ESAT6 & p15a & Prha & Rhamnose & cat & This study \\
\hline pLEMO-HbpD-ELL & p15a & Prha & Rhamnose & cat & This study \\
\hline pLEMO-HbpD-SpT2 & p15a & Prha & Rhamnose & cat & This study \\
\hline pLEMO-HbpD-SpT2-LL & p15a & Prha & Rhamnose & cat & This study \\
\hline pLEMO-HbpD-SpT2-Calm & p15a & Prha & Rhamnose & cat & This study \\
\hline pLEMO-HbpD-GFPnb & p15a & Prha & Rhamnose & cat & This study \\
\hline pLEMO-UpaG-SpT2-Calm & p15a & Prha & Rhamnose & cat & This study \\
\hline pLEMO-UpaG-GFPnb & p15a & Prha & rhamnose & cat & This study \\
\hline pRha-HbpD-ELL & pMB1 & Prha & Rhamnose & kan & This study \\
\hline pRha-HbpD-SpT2-Calm & pMB1 & Prha & Rhamnose & kan & This study \\
\hline pRha-HbpD-GFPnb & pMB1 & Prha & Rhamnose & kan & This study \\
\hline pTUM4 & p15a & Pcat & Constitutive & cat & {$[44]$} \\
\hline pTUM2 & p15a & Pcat & Constitutive & cat & This study \\
\hline pTUM6 & p15a & Pcat & Constitutive & cat & This study \\
\hline pET28-SpC2-mScarlet & pMB1 & PT7 & IPTG & kan & Lab collection \\
\hline pET20b-GFP-His6 & pMB1 & PT7 & IPTG & bla & $\begin{array}{l}\text { J.W. de Gier, } \\
\text { Stockholm, } \\
\text { Sweden }\end{array}$ \\
\hline pEH3-GFP & pMB1 & PlacUV5 & IPTG & cat & Lab collection \\
\hline
\end{tabular}

16), while the combined expression of the $\mathrm{HbpD}$ constructs and BAM yielded the highest induction of DegP. Co-expression of BAM did not appear to change the expression of HbpD-ESAT6 (Fig. 1B, D), indicating that optimal levels of this efficiently secreted construct were already reached in the presence of endogenous BAM. Importantly, however, production of HbpD-ELL markedly increased upon induction of the BAM complex from pJH114 (Fig. 1C, D), suggesting a favorable effect of extra BAM on the biogenesis of this difficult to secrete $\mathrm{HbpD}$ variant as hypothesized.

\section{Co-overexpression of the BAM complex improved surface display of HbpD-ELL and HbpD-SpT2-LL}

We next investigated whether the increased level of HbpD-ELL in the presence of overproduced BAM complex also reflects an increase in outer membrane translocation and, consequently, surface display of this construct. Display of HbpD-ELL and HbpD-ESAT6 was first assessed by direct whole-cell immune staining using flow cytometry. Cells were grown and induced for expression of the BAM complex and the HbpD variants and analysed for surface display using anti-ESAT6 monoclonal antibodies and flow cytometry (Fig. 2A). As expected, expression of HbpD-ESAT6 resulted in a clear shift in fluorescent intensity compared to noninduced cells. Co-overexpression of BAM did not further increase the fluorescent intensity, consistent with the Western blot analysis (Fig. 1). Cells only induced for HbpD-ELL expression resulted in a far less pronounced shift in fluorescent intensity (Fig. 2A). Cells induced for expression of both BAM and HbpD-ELL showed a clear increase in fluorescent intensity ( tenfold) compared to cells only induced for HbpD-ELL (Fig. 2B), indicating

\footnotetext{
(See figure on next page.)
}

Fig. 2 Co-overexpression of BAM improves surface display of HbpD-ELL. A Flow cytometric data showing the fluorescent intensity of cells bound by anti-ESAT6 monoclonal antibodies. The different culture conditions tested (i.e. HbpD-ESAT6 or HbpD-ELL uninduced, induced, or induced for BAM as well) are indicated on the right. B Fold change of surface display of cells expressing HbpD constructs as in (A). Graph shows the mean from three independent flow cytometry experiments. C Schematic representation and passenger models for HbpD-SpT2 and HbpD-SpT2-LL. Coupling of SpT2 to externally added SpC2-mScarlet allows detection of surface displayed HbpD-SpT2 and HbpD-SpT2-LL. D Coomassie-stained SDS-PAGE gels of whole cell lysates from E. coli BL21 (DE3) expressing HbpD-SpT2-LL with or without co-expression of BAM compared to lysates of cells expressing HbpD-SpT2 only. E Coomassie-stained SDS-PAGE gels of whole cell lysates from E. coli BL21 (DE3), expressing HbpD-SpT2 and HbpD-SpT2-LL and incubated with SpC2-mScarlet for $21 \mathrm{~h}$ at $4{ }^{\circ} \mathrm{C}$. HbpD variants and SpC2-mScarlet adducts $(<)$, BamA $(<)$ and non-bound SpC2-mScarlet $\left({ }^{*}\right)$ are indicated. Panel $\mathbf{E}$ is composed of lanes taken from the same image of a Coomassie-stained SDS-PAGE gel; the assembled parts are boxed 


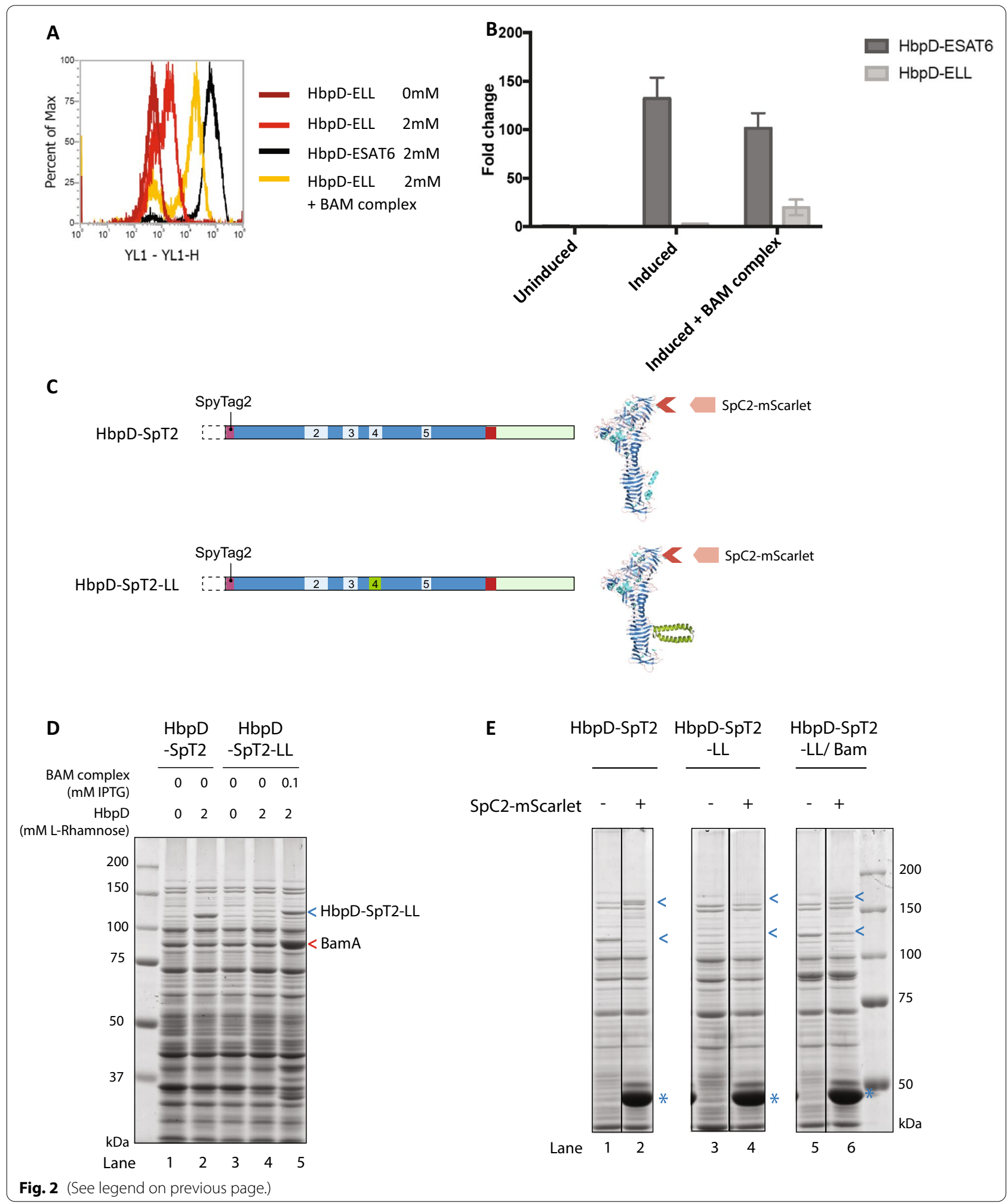

that increased expression of BAM, indeed, coincided with increased display of $\mathrm{HbpD}$-ELL at the cell surface (Fig. 2A, B). However, as also suggested by the Western blot data, the levels of surface-displayed HbpD-ELL did not reach those of the HbpD-ESAT6 control.

To confirm that the effect of bam co-overexpression on surface display was independent of antibody-based 
labelling, we used a direct labelling method that exploits the recently developed SpyTag/SpyCatcher protein ligation system [57]. In this system, a Spy-catcher domain $(\mathrm{SpC} 2)$ interacts with a Spy-tag peptide (SpT2) to form a covalent isopeptide bond. We exchanged the ESAT6 antigen in HbpD-ELL and HbpD-ESAT6 for SpT2, resulting in HbpD-SpT2-LL and HbpD-SpT2, respectively (Fig. 2C, Table 1). The constructs were cloned in a pLEMO plasmid under the control of Prha. Moreover, we fused the $\mathrm{SpC} 2$ domain to fluorescent protein $\mathrm{mScarlet}$ [1] yielding SpC2-mScarlet, which can be coupled to HbpD-SpT2 constructs displayed at the cell surface. As expected, DTS HbpD-SpT2-LL (121 kDa) was hardly detectable in BL21 (DE3) (Fig. 2D, lane 4) and detection improved upon co-expression of BAM (Fig. 2D, lane 5). To detect cellsurface exposure of HbpD-SpT2-LL and HbpD-SpT2, intact cells were incubated with purified $\mathrm{SpC} 2-\mathrm{mScarlet}$. Analysis by SDS-PAGE revealed a complete shift of the band representing HbpD-SpT2 to a higher position in the gel, suggesting that most of the protein was available for coupling and, hence, exposed at the cell surface (Fig. 2E, lanes 1-2). HbpD-SpT2-LL showed a similar shift in gel position when incubated with $\mathrm{SpC} 2-\mathrm{mScarlet}$, again indicative of cell-surface exposure (Fig. 2E, lanes 3-6). However, and in contrast to HbpD-SpT2, not all HbpDSpT2-LL expressed in presence of overproduced BAM shifted, indicating that not all HbpD-SpT2-LL was displayed at the cell surface. To verify that the shift in gel position was caused by coupling to $\mathrm{SpC} 2$-mScarlet we analyzed the samples by semi-native PAGE and imaged the gel for fluorescent protein bands. This confirmed the presence of fluorescent $\mathrm{SpC} 2-\mathrm{mScarlet}$-containing adducts coupled to HbpD-SpT2-LL (Additional file 1: Fig. S2). In conclusion, co-overexpression of the BAMcomplex resulted in improved expression and display of DTS HbpD-ELL and HbpD-SpT2-LL fusions at the cell-surface.

\section{Overexpression of the BAM complex improves secretion of two other DTS HbpD constructs}

Our results indicated a clear and positive effect of extra copies of the BAM complex on the expression and display of HbpD-ELL and HbpD-SpT2-LL. To investigate whether this is a generic effect, we tested the influence of co-expression of BAM on DTS HbpD constructs that carry either a calmodulin or a single-chain antibody domain. These segments were inserted at the $\mathrm{N}$-terminus of the passenger, where they replaced the protease subdomain of $\mathrm{Hbp}$ (Fig. 3; Table 1).

Calmodulin is a well-characterized domain of 145 amino acids (without any cysteines) that folds into a stable tertiary conformation in presence of $\mathrm{Ca}^{2+}$ ions $[16$, 58]. The $\mathrm{N}$-terminus of the calmodulin construct was extended with SpT2 and HA tags, yielding HbpD-SpT2Calm (Fig. 3A) and cloned into the pLEMO plasmid. Subsequently, E. coli BL21 (DE3) already carrying BAMexpression plasmid pJH114 was transformed with the pLEMO vector encoding HbpD-SpT2-Calm or HbpDSpT2 as control. The resulting co-transformants were grown in medium supplemented with $\mathrm{Ca}^{2+}$ to promote calmodulin folding and the same induction scheme was applied as outlined for BAM and HbpD-ELL above. Analysis of the samples in which BAM was not induced resulted in detection of the $\mathrm{HbpD}-\mathrm{SpT} 2$ at $\sim 117 \mathrm{kDa}$ upon, whereas DTS HbpD-SpT2-Calm migrated as a faint band at $\sim 138 \mathrm{kDa}$, suggesting inefficient secretion and concomitant degradation (Fig. 3B, lanes 2 and 4). However, BAM overexpression resulted in increased detection of HbpD-SpT2-Calm, reaching the level observed for the positive control HbpD-SpT2 (Fig. 3B, lane 6). To examine whether the improved expression levels coincided with improved cell-surface display, cells were incubated with $\mathrm{SpC} 2-\mathrm{mScarlet}$ and analyzed by flow cytometry (Fig. 3D). The results showed a low level of coupling of SpC2-mScarlet to un-induced cells and cells induced only for HbpD-SpT2-Calm. In contrast, co-expression of HbpD-SpT2-Calm with BAM led to an eight-fold increase of $\mathrm{SpC} 2$-mScarlet coupling, indicative of improved cell-surface display.

We then tested HbpD fused to a nanobody that binds GFP (GFPnb; Fig. 3A) [52]. Nanobodies are the antigenbinding fragments of the light chain of IgG antibodies (also known as $\left.\mathrm{V}_{\mathrm{HH}}\right)$. These small domains $(15 \mathrm{kDa})$ are known to fold in the periplasm and include one disulphide bond. Fusing GFPnb to HbpD has been shown to impair expression and surface display [14, 52]. We tested the effect of co-expression of BAM on expression and display of DTS HbpD-GFPnb cloned into pLEMO. Cells

(See figure on next page.)

Fig. 3 Co-overexpression of BAM improved the secretion of HbpD-Calm or HbpD-GFPnb chimeras. A Schematic representation and passenger models for HbpD-SpT2-Calm and HbpD-GFPnb. Coupling of SpC2-mScarlet allows detection of surface displayed HbpD-Spt2-Calm fusions, while binding of GFP to the GFPnb allows the detection of surface displayed HbpD-GFPnb. B, C Coomassie-stained SDS-PAGE gels with whole cell lysates of E. coli BL21 (DE3) cultures expressing HbpD chimeras. B Expression of HbpD-SpT2-Calm with and without overexpression of BAM compared to HbpD-SpT2. C Expression of HbpD-GFPnb with and without overexpression of BAM complex compared to HbpD-SpT2. D, E Fold change of fluorescence as detected by flow cytometry for binding of SpC2-mScarlet (D) or GFP (E) to HbpD-SpT2-Calm and HbpD-GFPnb when expressed with or without overproduced BAM complex. ${ }^{* *} \mathrm{p}<0.005$ 
A
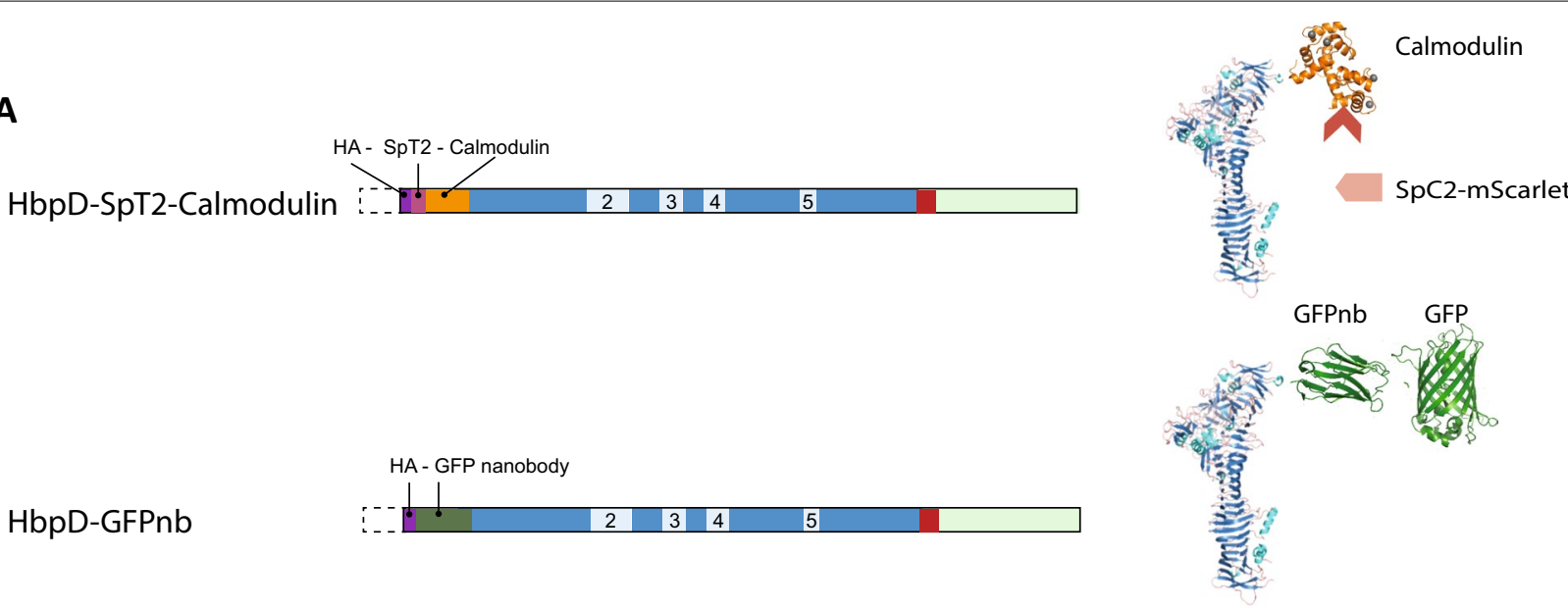

B

HbpD-GFPnb

B

\begin{tabular}{|c|c|c|c|c|c|c|}
\hline \multirow[b]{2}{*}{$\begin{array}{r}\text { BAM complex } \\
\text { (mM IPTG) }\end{array}$} & \multicolumn{2}{|c|}{ SpT2 } & \multicolumn{4}{|c|}{ SpT2-Calm } \\
\hline & 0 & 0 & 0 & 0 & 0.1 & 0.1 \\
\hline $\begin{array}{r}\text { HbpD } \\
\text { M L-Rhamnose) }\end{array}$ & 0 & 2 & 0 & 2 & 0 & 2 \\
\hline
\end{tabular}

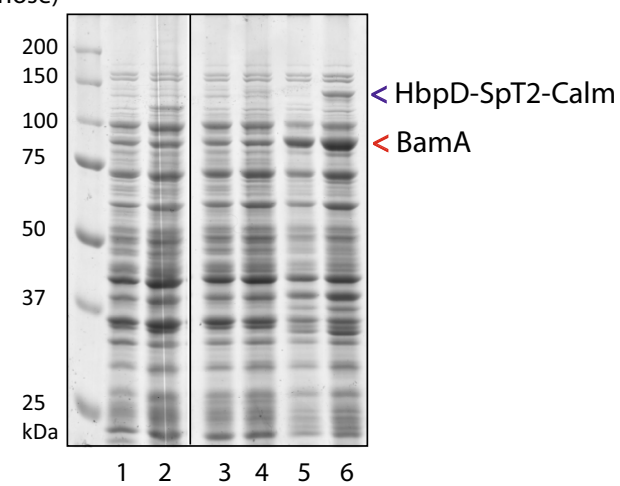

D

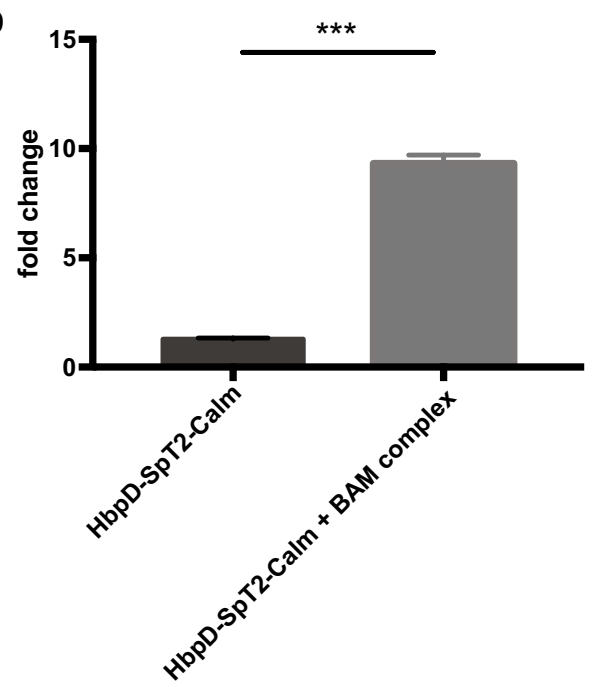

C

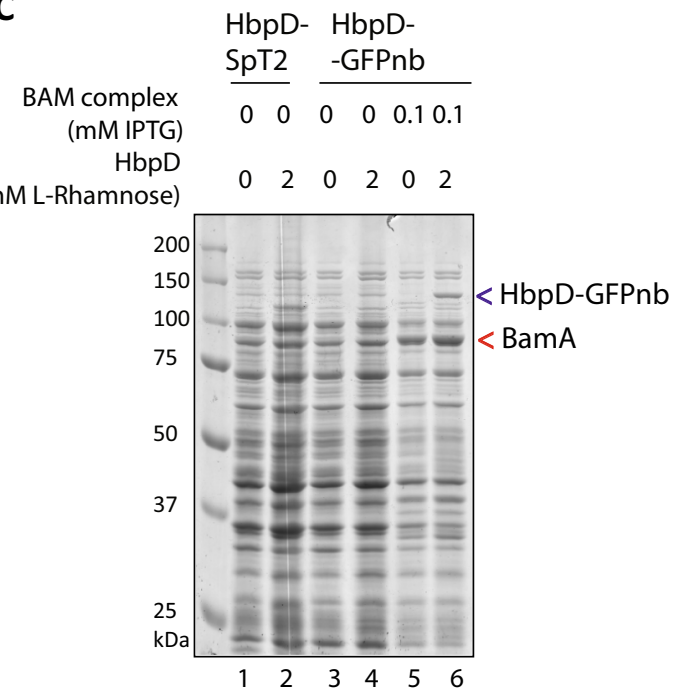

E

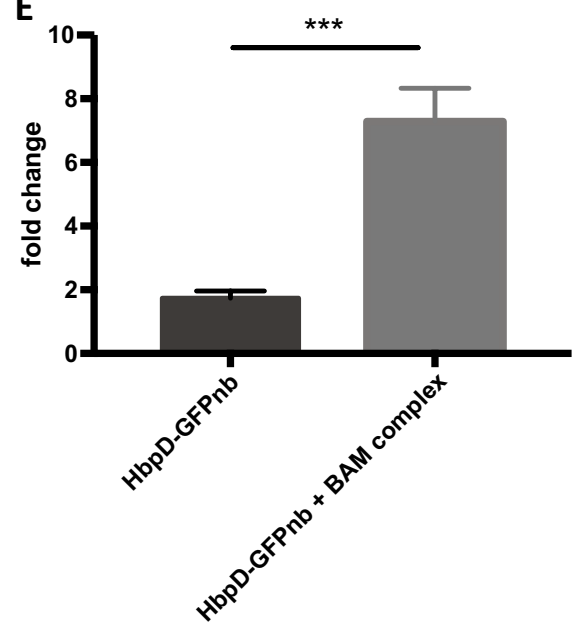

Fig. 3 (See legend on previous page.) 
not induced for extra BAM showed low expression levels of HbpD-GFPnb, when compared to the positive control HbpD-SpT2 (Fig. 3C, lane 2 and 4). However, expression of HbpD-GFPnb improved considerably in cells coexpressing BAM (Fig. 3C, lane 6). Improved cell-surface display was shown by incubating the cells with purified GFP followed by flow cytometry, which showed a clear shift of the fluorescence peak when BAM was overexpressed (Fig. 3E).

Taken together, overproduction of the BAM complex improves expression and surface display of three DTS variants, suggesting that BAM overexpression improves the tolerance of Hbp secretion for complex domains.

\section{Overexpression of the BAM complex supports display of a DTS trimeric autotransporter construct}

Not only the classical autotransporters, but also the other single-gene type $\mathrm{V}$ subclasses $(\mathrm{Vc}-\mathrm{Ve})$ require the BAM complex for translocation of their passengers to the cell surface [8]. We, therefore, investigated the effect of BAM overexpression on two DTS chimeras of a trimeric autotransporter (type Vc). Trimeric autotransporters share the domain organization of monomeric autotransporters, but their $\beta$-barrel and passenger domains assemble in trimers. We used a 170-residue truncate of UpaG, a trimeric autotransporter adhesin and known virulence factor of pathogenic E. coli $[45,51]$. We designed two potentially DTS UpaG variants with N-terminally either SpT2-Calmodulin (UpaG-SpT2-Calm; Fig. 4A; Table 1) or GFPnb (UpaG-GFPnb; Fig. 4B; Table 1). The pLEMO plasmids carrying these constructs were introduced in E. coli BL21 (DE3) harboring PJH114 and expression was tested as before.

When expression of the BAM complex was not induced, the two UpaG constructs were expressed to levels detectable on Western blot showing bands at $\sim 35 \mathrm{kDa}$ and $\sim 30 \mathrm{kDa}$ corresponding to the predicted masses calculated for monomeric UpaG-SpT2-Calm (38 kDa) and UpaG-GFPnb (32 kDa), respectively (Fig. 4A, B lane 2 ). Additional bands at higher positions in the gel could represent multimeric forms of the constructs, with the stronger reacting bands likely representing dimeric and multiple conformations of the trimeric form (Fig. 4A, B). Such a pattern was observed earlier for truncated UpaG [45] and trimeric autotransporter YadA [2]. Of note, trimeric autotransporters are known to be resistant to denaturation in SDS-PAGE [4]. Interestingly, expression of BAM from pJH114 together with the two DTS UpaG derivatives resulted in an increased intensity on gel of those bands that presumably represent dimeric and trimeric forms (Fig. 4A, B; lanes 4). Subsequent analysis by flow cytometry showed that co-expression of BAM resulted also in improved cell-surface exposure of UpaG-SpT2-Calm and UpaG-GFPnb (Fig. 4C, D). The addition of SpC2-mScarlet (Fig. 4C) or GFP (Fig. 4D) to cells co-expressing BAM and the two UpaG constructs yielded a significant increase in fluorescent signal compared to cells only induced for the UpaG constructs or non-induced cells. Apparently, the over-produced BAM complex improved cell surface display of both UpaG derivatives, suggesting that this was a rate-limiting step in the process. Overall, we conclude that the effect of BAM overproduction is likely generic for type $\mathrm{V}$ secretion systems.

\section{The influence of overproduction of periplasmic chaperones on DTS HbpD constructs}

Chaperones like DsbA, Skp, SurA and DegP keep secretion intermediates of autotransporters in a translocation competent state during their transit of the periplasm [54]. We, therefore, probed the effect of overproduction of periplasmic chaperones on the surface localization of the three DTS HbpD variants analysed here. Previous findings by the Skerra lab revealed that a combination of DsbA, DsbC, FkpA, Skp and SurA enhanced the expression of disulphide-bond containing proteins in $E$. coli [43]. DsbA and DsbC are thiol-disulfide oxidoreductases that catalyze the formation of disulfide bonds [25], FkpA functions as a general folding enhancer, while Skp and SurA are chaperones that escort $\beta$-barrel OMPs, including autotransporters, to the BAM complex $[10$, $23,26]$. To test the effect of extra copies of these chaperones on DTS HbpD constructs, we co-transformed BL21 (DE3) with a pTUM plasmid encoding chaperones and a pRHA plasmid encoding either HbpD-ELL, HbpD-SpT2-Calm, or HbpD-GFPnb (Table 1). The pTUM plasmids used were pTUM2 (encoding DsbA and DsbC), pTUM4 (encoding DsbA, DsbC, FkpA and SurA) and pTUM6 (encoding FkpA, SurA and Skp). While pTUM6 did not seem to have a highly significant beneficial effect, pTUM4 or pTUM2 improved expression of all three DTS HbpD derivatives albeit to different extents (Fig. 5B-D, lanes 3-5). The improved expression also led to an increase in cell surface display, as detected by flow cytometry (Fig. 5E-G). The fact that both pTUM2 and pTUM4 improved the expression of HbpD-ELL suggested that DsbA and DsbC affected the disulphide bond that is instrumental in blocking HbpD-ELL secretion [41]. On the other hand, presence of pTUM2 and pTUM4 also improved binding of $\mathrm{SpC} 2-\mathrm{mSc}$ carlet to $\mathrm{HbpD}-\mathrm{SpT} 2$ Calm and of GFP to HbpD-GFPnb (Fig. 5F, G), albeit with far lower fold-changes. The results are consistent with the reported effect of pTUM4 on expression of periplasmic proteins that lack disulfide bonds [44], and, therefore, may suggest that the slightly increased 


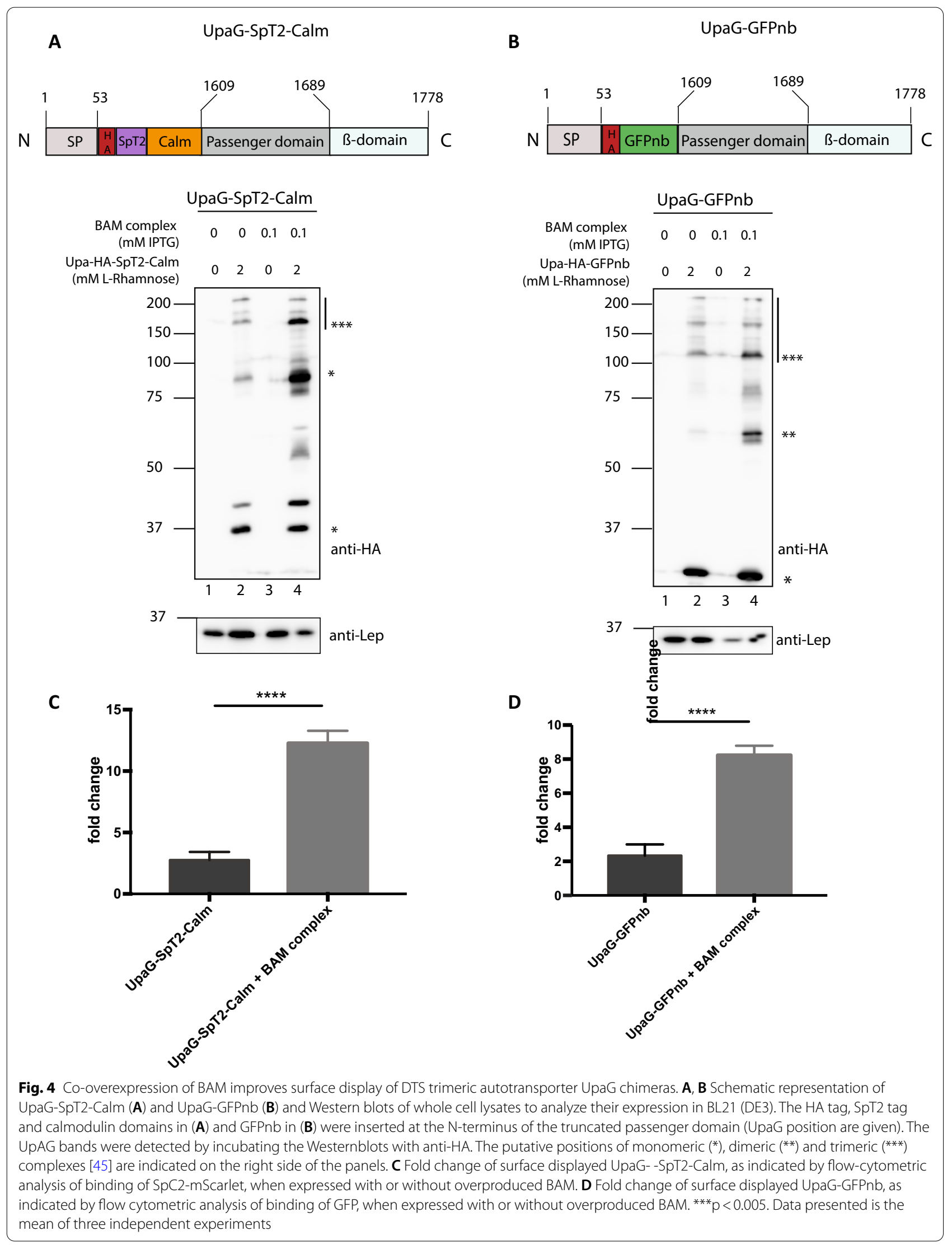




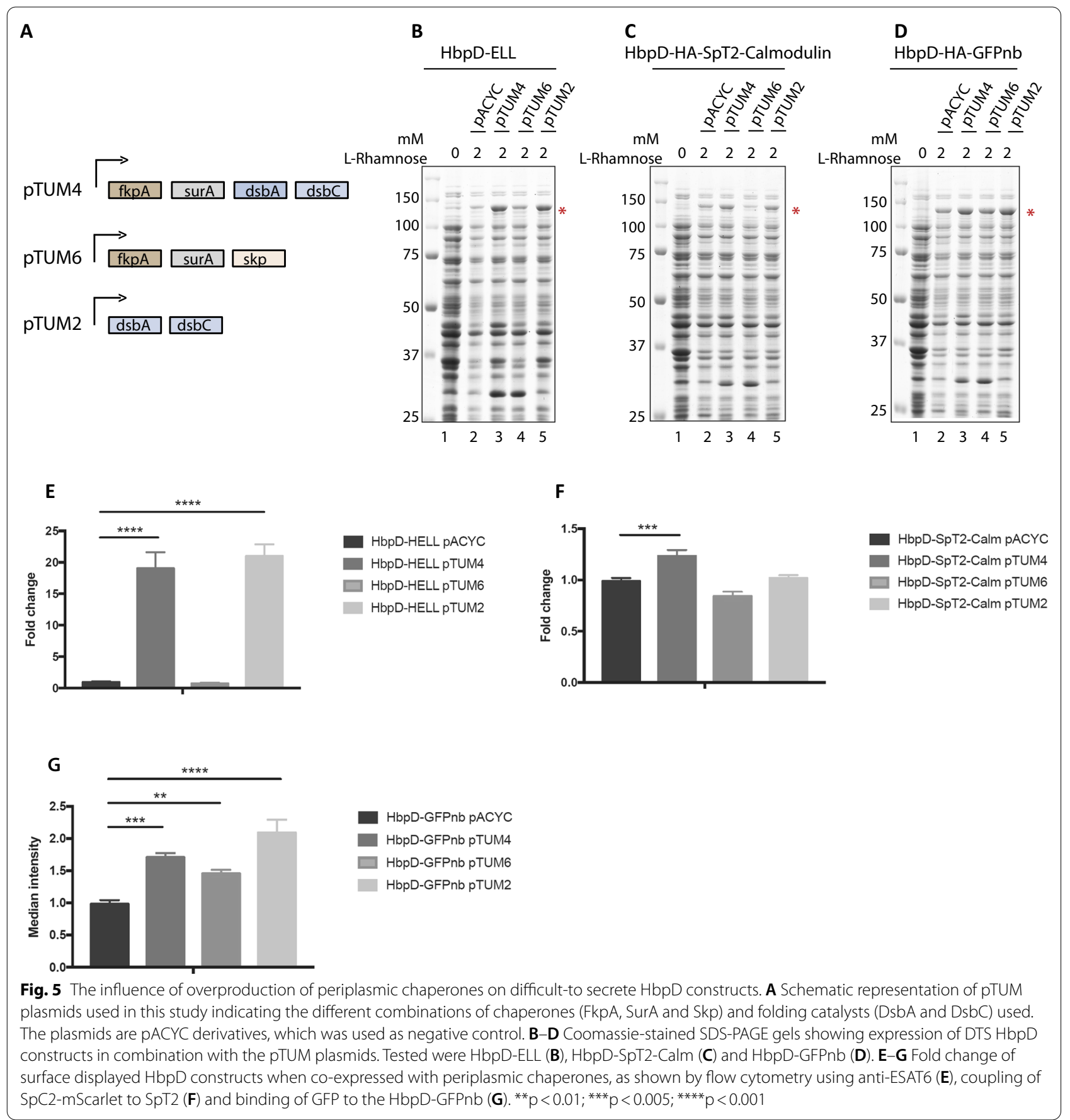

surface localization of DTS constructs tested here might be a direct or indirect consequence of the chaperones involved.

\section{Discussion}

Autotransporter-mediated surface display (also referred to as autodisplay) has been used in a variety of biotechnical applications including vaccine development, whole-cell biocatalysis, biosensor development, epitope mapping, and peptide library screening $[15,19,31]$. However, secretion of chimeric autotransporter constructs is limited by the size, structural complexity and folding propensity of the inserted cargo. In this study, we show that the secretion of recombinant DTS autotransporter chimeras can be improved by increasing the cellular concentration of the BAM complex and periplasmic chaperones. 
We opted to investigate these factors since our work had shown they associate with DTS derivatives of the model autotransporter $\mathrm{Hbp}$ that are jammed in the outer membrane due to engineered disulfide bonds or to insertion of folded protein segments $[14,16,40]$. Similarly, a mutation in the $\mathrm{C}$-terminal region of the passenger that is thought to nucleate its folding at the cell surface resulted in a stalled OM intermediate [47]. Finally, RNA sequencing showed that accumulation of a DTS Hbp mutant led to increased levels of mRNA encoding subunits of the BAM complex [48].

A plausible explanation for the beneficial effect of BAM overexpression is that translocation and OM-insertion of complex HbpD derivatives is slow, thus titrating available BAM complexes. This would trigger degradation in the periplasm by DegP and other proteases involved in cell envelope quality control $[16,48]$. Indeed, DTS Hbp derivatives induce extra-cellular stress responses and higher levels of the DegP proteases. BAM overexpression may provide more assembly sites for translocation but may also shield Hbp derivatives not yet at the cell surface from degradation in the periplasm. The latter explanation may be supported by the observation that externally adding SpC2-mScarlet to cells expressing DTS Hbp-SpT2 constructs did not result in full binding of these HbpDderivatives, while near-complete binding was observed for HbpD-SpT2 (Fig. 2). Furthermore, overexpression of BAM did not improve secretion of non-blocked variants of HbpD, like HbpD-ESAT6 or HbpD-SpT2, implying maximally efficient expression at endogenous levels of BAM. Similarly, increased expression of BAM did not influence the levels of other $\beta$-barrel OMPs, such as OmpA and the major porins OmpF and OmpC. A negative trade-off of co-expression of the BAM complex is a reduction in growth of the cultures (Additional file 1: Fig. S1) and an increase of cell-envelope stress as judged by the increase of DegP and SurA levels in cells when BAM is overexpressed (Fig. 1D). Nevertheless, vital processes, like Sec-mediated translocation across the cytoplasmic membrane, appeared not affected much, as indicated by the absence of accumulation of unprocessed SurA, a soluble periplasmic protein transported by the Sec translocon (Fig. 1D).

We initially focused on the monomeric autotransporter Hbp, but BAM co-expression also improved the secretion of trimeric autotransporter UpaG chimeras. Possibly, other $\beta$-barrel proteins that are difficult to assemble in the outer membrane could also benefit from the presence of extra copies of the BAM complex, which may expand its use for biotechnical applications. For example, the inverse autotransporters (subclass type $\mathrm{Ve}$ ) have also been used for surface display [38]. An alternative strategy to BAM co-expression could be to lower the amount of substrates for endogenous BAM. This has been achieved by deleting genes encoding or regulating OMPs, yielding strains that showed improved expression of recombinant OMPs and trimeric autotransporter constructs [29, 34]. However, in our hands the strain of Prilipov et al. did not yield the levels of HbpD-ELL obtained when co-expressing BAM (results not shown).

In comparison to the generic effect of co-expressing $\mathrm{BAM}$, the effect of co-expressing periplasmic chaperones and folding catalysts [44] appeared variable and limited (Fig. 5). The major effect observed, a positive effect of DsbA/C on HbpD-ELL expression and display, can very likely be attributed to the release of the secretion-blocking disulphide bond in the construct [41]. Apparently, overproduction of both DsbA and DsbC results not only in increased formation of disulphide bonds, but also in increased reduction of these bonds, causing modulation between stalled and secretion-competent states. However, we cannot rule out an indirect effect of DsbA/DsbC on DTS Hbp constructs, in view of the observed effects on the chimeras that lack cysteines. In contrast, overexpression of SurA and Skp, which have been reported to interact with nascent autotransporters in the periplasm $[12,40,47]$ showed only a limited influence.

Our earlier unsuccessful attempts to improve the secretion of DTS Hbp chimeras focused on modifying the $\beta$-barrel at the $C$-terminus. These approaches included inserting extra $\beta$-hairpins to enlarge the $\beta$-barrel channel [39], or replacing the $\beta$-barrel for the larger $\beta$-barrel of OMP FhuA (unpublished data). However, the interactions between passenger, $\beta$-barrel domain and BAM complex may be too fine-tuned to tolerate such adaptations. A simple replacement of the Hbp $\beta$-domain for that of the nearly identical autotransporter EspP already caused a reduction in secretion efficiency of the $\mathrm{Hbp}$ [39]. It is also clear from our work and that of many others, that the choice of $\beta$-barrel domain and the site where recombinant proteins are fused to the $\beta$-barrel or inserted within the passenger domain may influence secretion efficiency and requires testing of different possibilities $[14,35]$. The results presented here suggest that co-expression of the BAM complex offers a straightforward and generic solution for impeded autotransporter secretion, probably by providing an extended time-window for the secretion process. It remains to be shown whether it is also a solution to improve the expression of other recombinant $\beta$-barrel proteins.

\section{Materials and methods}

Strains and growth conditions

E. coli strain Top 10F' (Invitrogen, UK) was used for cloning and BL21(DE3) (Novagen, Germany) was used for expression experiments. Both were grown in lysogeny 
broth (LB; $10 \mathrm{~g} / \mathrm{L}$ tryptone, $5 \mathrm{~g} / \mathrm{L}$ yeast extract, $10 \mathrm{~g} / \mathrm{L}$ $\mathrm{NaCl}$ ). For expression experiments the LB was supplemented with $0.2 \%(\mathrm{w} / \mathrm{v})$ glycerol. To select for plasmids (Table 2) antibiotics were added to the following concentrations: ampicillin, $100 \mu \mathrm{g} / \mathrm{mL}$; kanamycin, $50 \mu \mathrm{g} / \mathrm{mL}$; and chloramphenicol $30 \mu \mathrm{g} / \mathrm{mL}$. Unless stated otherwise, cultures were incubated at $37^{\circ} \mathrm{C}$ with shaking.

\section{Reagents, chemicals, enzymes and sera}

Rapid DNA De-phosphorylation and Ligation Kit was obtained from Roche Applied Science, restriction enzymes and Phusion High Fidelity DNA polymerase from New England Biolabs. The pre-stained Precision Plus SDS-PAGE protein marker was obtained from Biorad. Sigma-Aldrich provided all other reagents, primers and chemicals. 96-well plates used were $\mu$ Clear Chimney black clear-bottom plates from Greiner. Immunostaining was performed with mouse monoclonal antibodies directed against HA, ESAT6 (Hyb 76-8) [21] or with rabbit polyclonal serum recognizing the Hbp $\beta$-barrel (SN477) [53], BamA (a kind gift of J. Tommassen, Utrecht University, The Netherlands), SurA (a kind gift of T. Silhavy, Princeton University, USA), leader peptidase (Lep; from our own laboratory collection), or DegP (a kind gift of J. Beckwith, Harvard Medical School, USA). Secondary antisera used were peroxidase-conjugated goat-anti-mouse and goat-anti-rabbit IgGs (Rockland Immunochemicals). Lumi-Light Western Blotting Substrate was obtained from Roche, skim milk from Thermo Fisher Scientific. All synthetic DNA constructs were obtained using GeneArt (Thermo Scientific).

\section{Expression plasmids}

The BAM complex was expressed from plasmid pJH114 which carries the five genes $\operatorname{bam} A-E$ cloned into an operon under the control of an IPTG-inducible trc promoter [37]. This plasmid was co-transformed with pLEMO [55] and pRha-derived [7] plasmids (a kind gift of J.W de Gier, Stockholm University, Sweden) carrying the regulatory cassette enabling the rhamnose-mediated induction of Prha and the HbpD-constructs described in Table 2 under control of this promoter. All constructs have been confirmed by sequencing (Macrogen). The plasmids encoding the chimeras were constructed as follows:

\section{HbpD-ESAT6 and HbpD-ELL constructs}

Fragments including the ORFS encoding HbpD-ESAT6 were obtained by was produced by PCR using pEH3HbpD-ESAT6 plasmid [18] and pEH3-HbpD-ELL [41] as a template and primers Fw-EcoRI-SalI-HbpD (5-CGA ATTCGTCGACACCATGAACAGAATTTATTC TCT TCGC-3) and Rv-HbpD-BamHI-HindIII (5'-CCA
AGCTTGGATCCTCAGAATGAATAACGAATATTA GCG-3). The PCR products were digested with Sall and BamHI and ligated into pLemo plasmid digested with the same enzymes. The corresponding pRha-HbpD-ELL plasmid was also made by PCR using the same template and forward primer in combination with primer HindIIIHbp-Rv (5'-CTGAAAGCTTCAGAATGAATAACGAAT ATTAGCG-3) and cloned into the pRha vector using a SalI-HinDIII fragment.

\section{HbpD-SpT2 and HbpD-SpT2-L9L9}

To allow for direct labelling of Hbp chimeras at the cell surface we used the SpyTag/SpyCatcher protein ligation system [57]. In this system, a Spy-catcher domain interacts with a Spy-tag peptide to form a covalent isopeptide bond. An optimized version of the tag, SpT2 [20] was cloned into $h b p$ to replace the ESAT6 antigen, resulting in $\mathrm{HbpD}-\mathrm{SpT} 2-\mathrm{LL}$ and $\mathrm{HbpD}-\mathrm{SpT} 2$, respectively (Fig. 2C). For cloning a synthetic DNA fragment including restriction sites SacI and EagI was ordered and subsequently cloned into pLemo-HbpD-ELL and pLemoHbpD-ESAT6 cut with the same enzymes.

\section{HbpD-SpT2-Calm and HbpD-GFPnb}

A synthetic DNA fragment encoding HA-SpT2-Calm was cloned into an pEH3-HbpD construct [18] by in-fusion cloning (Invitrogen) using the SacI/BamHI restriction sites present in that plasmid resulting in $\mathrm{pEH} 3-\mathrm{HbpD}$ SpT2-Calm. Next, a SacI-KpnI fragment from this plasmid was inserted into pLEMO-HbpD-ESAT6 plasmid digested with the same enzymes, resulting in pLemoHbpD-SpT2-Calm. Following the same procedure, a synthetic DNA fragment encoding HA-GFPnb was ordered and inserted into the pLemo-HbpD-ESAT6 plasmid to yield pLemo-HbpD-GFPnb. The pRha plasmids encoding HbpD-SpT2-Calm and HbpD-GFPnb were subsequently made by replacing the SacI-KpnI fragment of pRhaHbpD-ELL by a SacI-KpnI fragment of pLemo-HbpDSpT2-Calm and pLemo-HbpD-GFPnb, respectively.

\section{UpaG-SpT2-Calm and UpaG-GFPnb}

Expression of full-length (1778 residue) UpaG protein was reported to be toxic in $E$. coli $\mathrm{K} 12$, but a 170 -residues truncate of UpaG that contains the signal peptide and $\sim 80$ residues of the native-passenger domain did not affect growth [46] and an N-terminal fusion of the SpT2 tag resulted in its exposure on the cell surface [45]. We used this information to design two potentially DTS variants of the truncated UpaG, UpaG-SpT2-Calm (Fig. 4A) and UpaG-GFPnb (Fig. 4B). A $\sim 1.1 \mathrm{~kb}$ synthetic DNA encoding UpaG-SpC2 including restriction sites for EcoRI and HindIII was cloned into the HinDIII and EcoRI sites of pRha by Gibson assembly [28]. Subsequently, a 
1.3 Kbp synthetic encoding HA-SpT2-Calm was cloned into the $\mathrm{XbaI}$ and EcoRI sites, to replace the HA-SpC2 encoding part, yielding pRha-UpaG-SpT2-Calm. Then, the ORF encoding UpaG-SpT2-Calmodulin fragment was amplified by PCR using pRha-med-UpaG-SpT2Calm as template and primers c4424_SalI_FW (5'-CGT CGACATGAACAAAATCTTCAAAGTAATCTGGAA CC-3) and c4424_AvrII_Rv (5'-CCTAGGTTACCACTG GATACCTGCCC-3). The PCR product was digested with SalI and AvrII and ligated into the pLemo plasmid digested with the same enzymes generating pLemoUpaG-SpT2-Calmodulin. Similarly, a synthetic 391-bp HA-GFPnb-encoding DNA fragment was introduced into pRha-med-UpaG-SpT2-Calmodulin to replace HASpT2-Calmodulin using HindIII and BamHI. The ORF encoding UpaG-GFPnb was then amplified by PCR using pRha-med-UpaG-GFPnb as a template and primer pair c4424_SalI_FW and c4424_AvrII_Rv. The PCR product was then digested with SalI and AvrII to be introduced into the pLemo plasmid cut with the same enzymes generating pLemo-UpaG-GFPnb.

\section{Other plasmids}

The ORF encoding the fusion of $\mathrm{SpC} 2[20]$ and $\mathrm{mScarlet}$ [1] was based upon the published sequences and cloned into the NcoI and HinDIII restriction sites of the pET28a plasmid (Novagen). The ORFs encoding FkpA and SurA were deleted from pTUM4 (a kind gift of A. Skerra, Technische Universität München, Germany) to yield pTUM2, encoding the DsbA and DsbC proteins. To construct pTUM6, first the $d s b A$ and $d s b C$ genes were deleted from pTUM4 and the ORF encoding the Skp gene was inserted.

\section{Growth and protein expression in 96-well plates}

Growth and expression assays were performed in 96-well plates. Bacteria were grown in regular culture flasks in LB medium containing $0.2 \%$ glycerol to mid-log phase. Subsequently, the culture was diluted to an optical density at $600 \mathrm{~nm}(\mathrm{OD} 600)$ of 0.2 and $200 \mu \mathrm{L}$ was transferred to a 96-well plate and growth was continued for $1 \mathrm{~h}$. First, expression of the BAM complex was induced by adding IPTG to a final concentration of $100 \mu \mathrm{M}$. Growth was prolonged for $1 \mathrm{~h}$, followed by the induction of genes encoded on the pLemo/pRha plasmids by adding L-Rhamnose to a final concentration of $2 \mathrm{mM}$, after which growth was continued for $2 \mathrm{~h}$. During growth, plates were sealed and placed in Thermostar (BMG Labtech) shakers at 600 RPM. The OD600 was measured using the HTX Synergy plate-reader (BMG Labtech). After $2 \mathrm{~h}$ incubation with $2 \mathrm{mM} \mathrm{L}$-Rhamnose, culture samples were taken by mixing the cultures with an equal volume of two-times concentrated sample buffer. Samples were then boiled for
10 min and run on SDS-PAGE gels. The gels were either stained with Coomassie Brilliant Blue or subjected to immune-blotting as described earlier [17].

\section{ESAT6-based flow cytometry}

Bacterial cultures were started at an OD of 0.05 . When cultures reached OD600 of 0.6, IPTG was added to a final concentration of $0.1 \mathrm{mM}$ and grown for $1 \mathrm{~h}$. Then, L-Rhamnose was added to a final concentration of $2 \mathrm{mM}$ and incubation was prolonged for $2 \mathrm{~h}$. In separate cultures, E. coli BL21 DE3 carrying pEH3-GFP [52] was grown and IPTG was added to a final concentration of $1 \mathrm{mM}$ to induce GFP production. After induction, the cultures were grown for $2 \mathrm{~h}$. After culturing, 0.02 OD600 units of $E$. coli cultures expressing the DTS constructs, or their controls, to be tested were mixed with 0.18 OD600 units of $E$. coli expressing GFP. The GFP-expressing bacteria were added to obtain a sufficient number of cells, while reducing the amount of anti-ESAT6 antiserum needed for efficient labeling. The mixtures were harvested by centrifugation at $4{ }^{\circ} \mathrm{C}$ and $9000 \times g$ for $5 \mathrm{~min}$., washed once with PBS (pH 7.4) and then resuspended in PBS containing $0.7 \%$ poly-formaldehyde (PFA) and fixed overnight at $4{ }^{\circ} \mathrm{C}$. PFA was washed away by centrifuging two times, resuspending the fixed cells in PBS. After this, pellets were resuspended in $50 \mu \mathrm{L}$ PBS $1 \%$ BSA containing a 1:25 dilution of ESAT6 (Hyb 76-8), incubated for $1 \mathrm{~h}$ at room temperature, followed by 3 times of washing with $750 \mu \mathrm{L}$ PBS 1\% BSA/0.05\% Tween 20 . Next, the pellets were resuspended in $50 \mu \mathrm{L}$ PBS $1 \%$ BSA containing a 1:50 dilution of the Goat anti-mouse antiserum conjugated to Alexa Dye 568 and 1:1000 dilution of SYTO63 to stain the bacterial DNA. After incubation for $1 \mathrm{~h}$ at room temperature, the samples were washed three times with $750 \mu \mathrm{L}$ PBS $1 \%$ BSA $/ 0.05 \%$ Tween 20 . Finally, pellets were resuspended in $1 \mathrm{~mL}$ PBS $1 \%$ BSA and subjected to flow-cytometric analysis on an Attune NxT flow cytometer (ThermoFisher). Bacteria were gated based on the DNA stain SYTO63 (RL1-670/14 nm). GFP expression was detected in gate BL1-530/30 nm and ESAT6 (through antibody-bound Alexa Dye 568) was detected in YL1-585/16 nm. The fluorescence on the cells of interest (SYTO63+, GFP-) was quantified and compared to cells stained for secondary antibody only.

\section{Purification of SpC2-mScarlet and GFP-His6}

E. coli $\mathrm{BL} 21(\mathrm{DE} 3)$ cells harboring $\mathrm{pET} 28-\mathrm{SpC} 2-\mathrm{mScar}-$ let or pET20b-GFP-His6 were grown in LB containing $0.2 \%$ glucose to an OD600 of 0.4-0.5. IPTG was added to a final concentration of $1 \mathrm{mM}$, after which cultures were grown for another $2-4 \mathrm{~h}$. Cells were harvested by centrifugation, washed with $\mathrm{PBS}(\mathrm{pH}$ 7.4) then resuspended in buffer $\mathrm{A}\left(50 \mathrm{mM} \mathrm{Na}{ }_{3} \mathrm{PO}_{4}, 300 \mathrm{mM} \mathrm{NaCl}[\mathrm{pH}\right.$ 
7.4]). Phenylmethylsulfonyl fluoride (PMSF) was added to a concentration of $125 \mu \mathrm{M}$. The cells were then disrupted by two passages through a One Shot cell disruptor (Constant Systems Ltd., UK) set at 1.2 Pa. Cell debris was removed by centrifugation at $4{ }^{\circ} \mathrm{C}$ and $10,000 \times g$ and membrane fragments were removed by centrifugation at $4{ }^{\circ} \mathrm{C}$ and $293,000 \times g$, respectively. The His6-tagged proteins were then purified from the supernatant using Talon Superflow medium (GE Healthcare Life Sciences) according to the manufacturer's instructions. Eluates were dialyzed overnight at $4{ }^{\circ} \mathrm{C}$ against up to 1000 volumes of PBS (pH 7.4). After dialysis, glycerol was added to $10 \%$, and aliquots were stored at $-80{ }^{\circ} \mathrm{C}$.

\section{Spy ligation of $\mathrm{SpC2}$-mScarlet in cells}

To 0.5 OD600 units of $E$. coli BL21 DE3 cells expressing either the BAM complex, the SpT2 HbpD constructs, or both, $5 \mu \mathrm{L}$ of purified $\mathrm{SpC} 2$-mscarlet $(9.2 \mathrm{mg} / \mathrm{mL})$ was added. The mixtures were incubated overnight at $4{ }^{\circ} \mathrm{C}$ to allow protein ligation to occur. The cells were harvested by centrifugation and washed with PBS containing $0.05 \%$ Tween 20 and fixed in 2\% PFA in PBS for $15 \mathrm{~min}$, followed by three times of washing with $750 \mu \mathrm{L}$ PBS containing $0.05 \%$ Tween 20 . The mixtures were then incubated with Syto62 (1:1000 dilution) for $10 \mathrm{~min}$, washed with PBS containing 0.05\% Tween 20 and analyzed by flow-cytometric analysis as described above. For SDS-PAGE, equal volumes of cells were mixed with $2 \times$ sample buffer and boiled for gels stained by Coomassie Brilliant blue. To analyse fluorescence, semi-native SDSPAGE was performed, by omitting the SDS was omitted from the gels, but not from the running buffer and loading of non-boiled samples. After electrophoresis, the gels were immediately imaged for GFP fluorescence using an AI600 imager (Amersham).

\section{GFP binding to GFPnb on cell surface}

To 0.5 OD of E. coli BL21 DE3 strains expressing either the BAM complex, the DTS GFPnb constructs, or both $1.63 \mu \mathrm{L}$ of purified GFP $(92 \mu \mathrm{M})$ was added. After $15 \mathrm{~min}$ of incubation on ice, the mixtures were spin down for $5 \mathrm{~min}$ at $5000 \mathrm{rpm}$ and washed 3 times with PBS containing $0.05 \%$ Tween 20 . Next, the mixtures were incubated with Syto9 (1:1000 dilution) for $10 \mathrm{~min}$, then washed with PBS containing $0.05 \%$ Tween 20 and analyzed by flowcytometric analysis. Samples were also mixed with $2 \times$ sample buffer and subjected to SDS-PAGE and staining with Coomassie Brilliant blue. To analyse fluorescence, semi-native SDS-PAGE was performed as described above.

\section{Abbreviations}

BAM: $\beta$-Barrel assembly machinery; OM: Outer membrane; OMP: Outer membrane protein; Hbp: Hemoglobin protease; OMVs: Outer membrane vesicles; HbpD: Hbp Display constructs; DTS: Difficult-to-secrete; IPTG: Isopropyl $\beta$-D-1thiogalactopyranoside; Calm: Calmodulin; SpT2: Spy tag 2; SpC2: Spy catcher 2; HA: Haemagglutinin; GFP: Green fluorescent protein; GFPnb: GFP-recognizing nanobody; PMSF: Phenylmethylsulfonyl.

\section{Supplementary Information}

The online version contains supplementary material available at https://doi. org/10.1186/s12934-021-01668-2.

Additional file 1: Figure S1. Plot of the growth curves of BL21 DE3 expressing either HbpD-ESAT6, HbpD-ELL alone or together with BAM from $\mathrm{pJH114}$. Figure S2. Fluorescence image of a semi-native SDS-PAGE gel with samples of cultures co-expressing HbpD-SpT2, HbpD-SpT2-LL or HbpD-SpT2-LL with BAM that were incubated with SpC2-mScarlet or not to allow for coupling of the fluorescent protein to the HbpD-SpT2 variants exposed on the cell surface.

\section{Acknowledgements}

We thank Corinne M Ten Hagen-Jongman for construction of the pTUM plasmids and Mariah Kes for initial experiments on the BAM complex.

\section{Funding}

TP was funded by the BAC-Vactory program, domain of Applied and Engineering Sciences (TTW) of the Netherlands Organization for Scientific Research (NWO). DH received funding from the European Union's Horizon 2020 Research and Innovation Program under the Marie Skłodowska-Curie Grant agreement No. 812915.

\section{Declarations}

\section{Competing interests}

\lrcorner and WJ are involved in Abera Bioscience AB that aims to exploit the presented technology. The remaining authors declare that the research was conducted in the absence of any commercial or financial relationships that could be construed as a potential conflict of interest.

\section{Author details \\ ${ }^{1}$ Department of Molecular Microbiology, Amsterdam Institute of Molecular and Life Sciences, Vrije Universiteit Amsterdam, Amsterdam, The Netherlands. ${ }^{2}$ Medical Microbiology and Infection Control, Amsterdam Institute of Infection \& Immunity, Amsterdam UMC, Amsterdam, The Netherlands. ${ }^{3}$ Abera Biosci- ence AB, Solna, Sweden.}

Received: 5 May 2021 Accepted: 26 August 2021

Published online: 06 September 2021

\section{References}

1. Bindels DS, Haarbosch L, van Weeren L, Postma M, Wiese KE, Mastop M, Aumonier S, Gotthard G, Royant A, Hink MA, Gadella TW Jr. mScarlet: a bright monomeric red fluorescent protein for cellular imaging. Nat Methods. 2017;14:53-6.

2. Chauhan N, Hatlem D, Orwick-Rydmark M, Schneider K, Floetenmeyer M, van Rossum B, Leo JC, Linke D. Insights into the autotransport process of a trimeric autotransporter, Yersinia Adhesin A (YadA). Mol Microbiol. 2019;111(3):844-62. https://doi.org/10.1111/mmi.14195 (Epub 2019 Feb 13).

3. Christie PJ. The rich tapestry of bacterial protein translocation systems. Protein J. 2019;38:389-408.

4. Cotter SE, Surana NK, Grass S, St Geme JW. Trimeric autotransporters require trimerization of the passenger domain for stability and adhesive activity. J Bacteriol. 2006;188:5400-7. 
5. Daleke-Schermerhorn MH, Felix T, Soprova Z, Ten Hagen-Jongman CM, Vikstrom D, Majlessi L, Beskers J, Follmann F, de Punder K, van der Wel NN, Baumgarten T, Pham TV, Piersma SR, Jimenez CR, van Ulsen P, de Gier JW, Leclerc C, Jong WS, Luirink J. Decoration of outer membrane vesicles with multiple antigens by using an autotransporter approach. Appl Environ Microbiol. 2014;80:5854-65.

6. Doyle MT, Bernstein HD. BamA forms a translocation channel for polypeptide export across the bacterial outer membrane. Mol Cell. 2021;81(9):2000-2012.e3. https://doi.org/10.1016/..molcel.2021.02.023.

7. Giacalone MJ, Gentile AM, Lovitt BT, Berkley NL, Gunderson CW, Surber MW. Toxic protein expression in Escherichia coli using a rhamnosebased tightly regulated and tunable promoter system. Biotechniques. 2006;40(3):355-64. https://doi.org/10.2144/000112112.

8. Grijpstra J, Arenas J, Rutten L, Tommassen J. Autotransporter secretion: varying on a theme. Res Microbiol. 2013;164:562-82.

9. Hart EM, Mitchell AM, Konovalova A, Grabowicz M, Sheng J, Han X, Rodriguez-Rivera FP, Schwaid AG, Malinverni JC, Balibar CJ, Bodea S, Si Q, Wang H, Homsher MF, Painter RE, Ogawa AK, Sutterlin H, Roemer T, Black TA, Rothman DM, Walker SS, Silhavy TJ. A small-molecule inhibitor of BamA impervious to efflux and the outer membrane permeability barrier. Proc Natl Acad Sci USA. 2019;116:21748-57.

10. Hennecke G, Nolte J, Volkmer-Engert R, Schneider-Mergener J, Behrens $\mathrm{S}$. The periplasmic chaperone SurA exploits two features characteristic of integral outer membrane proteins for selective substrate recognition. J Biol Chem. 2005;280:23540-8.

11. leva R, Bernstein HD. Interaction of an autotransporter passenger domain with BamA during its translocation across the bacterial outer membrane. Proc Natl Acad Sci USA. 2009;106:19120-5.

12. leva R, Tian P, Peterson JH, Bernstein HD. Sequential and spatially restricted interactions of assembly factors with an autotransporter beta domain. Proc Natl Acad Sci USA. 2011;108:E383-91.

13. Jong WS, Daleke-Schermerhorn MH, Vikström D, Ten Hagen-Jongman CM, de Punder K, van der Wel NN, van de Sandt CE, Rimmelzwaan GF, Follmann F, Agger EM, Andersen P, de Gier JW, Luirink J. An autotransporter display platform for the development of multivalent recombinant bacterial vector vaccines. Microb Cell Fact. 2014;13:162.

14. Jong WSP, Schillemans M, Ten Hagen-Jongman CM, Luirink J, van Ulsen P. Comparing autotransporter beta-domain configurations for their capacity to secrete heterologous proteins to the cell surface. PLOS ONE. 2018;13:e0191622.

15. Jong WS, Sauri A, Luirink J. Extracellular production of recombinant proteins using bacterial autotransporters. Curr Opin Biotechnol. 2010;21:646-52.

16. Jong WS, ten Hagen-Jongman CM, den Blaauwen T, Slotboom DJ, Tame JR, Wickstrom D, de Gier JW, Otto BR, Luirink J. Limited tolerance towards folded elements during secretion of the autotransporter Hbp. Mol Microbiol. 2007;63:1524-36.

17. Jong WS, Vikstrom D, Houben D, van den Berg van Saparoea HB, de Gier JW, Luirink J. Application of an E. coli signal sequence as a versatile inclusion body tag. Microb Cell Fact. 2017;16:50.

18. Jong WSP, Soprova Z, de Punder K, ten Hagen-Jongman CM, Wagner S, Wickström D, de Gier J-W, Andersen P, van der Wel NN, Luirink J. A structurally informed autotransporter platform for efficient heterologous protein secretion and display. Microb Cell Fact. 2012;11:85.

19. Jose J, Meyer TF. The autodisplay story, from discovery to biotechnical and biomedical applications. Microbiol Mol Biol Rev. 2007;71:600-19.

20. Keeble AH, Banerjee A, Ferla MP, Reddington SC, Anuar I, Howarth M. Evolving accelerated amidation by SpyTag/SpyCatcher to analyze membrane dynamics. Angew Chem Int Ed Engl. 2017;56:16521-5.

21. Klausen J, Magnusson M, Bengård Andersen Å, Koch C. Characterization of purified protein derivative of tuberculin by use of monoclonal antibodies: isolation of a delayed-type hypersensitivity reactive component from M. tuberculosis culture filtrate. Scand J Immunol. 1994;40:345-9.

22. Konovalova A, Kahne DE, Silhavy TJ. Outer membrane biogenesis. Annu Rev Microbiol. 2017;71:539-56.

23. Korndörfer IP, Dommel MK, Skerra A. Structure of the periplasmic chaperone Skp suggests functional similarity with cytosolic chaperones despite differing architecture. Nat Struct Mol Biol. 2004;11:1015-20.

24. Kuipers G, Karyolaimos A, Zhang Z, Ismail N, Trinco G, Vikstrom D, Slotboom DJ, de Gier JW. The tunable pReX expression vector enables optimizing the T7-based production of membrane and secretory proteins in E. coli. Microb Cell Fact. 2017;16:226.

25. Kurokawa $Y$, Yanagi $H$, Yura T. Overexpression of protein disulfide isomerase DsbC stabilizes multiple-disulfide-bonded recombinant protein produced and transported to the periplasm in Escherichia coli. Appl Environ Microbiol. 2000;66:3960-5.

26. Lazar SW, Kolter R. SurA assists the folding of Escherichia coli outer membrane proteins. J Bacteriol. 1996;178:1770-3.

27. Lee J, Tomasek D, Santos TM, May MD, Meuskens I, Kahne D. Formation of a $\beta$-barrel membrane protein is catalyzed by the interior surface of the assembly machine protein BamA. Elife. 2019;8:e49787. https://doi.org/10. 7554/eLife.49787.

28. Merryman C, Gibson DG. Methods and applications for assembling large DNA constructs. Metab Eng. 2012;14:196-204.

29. Meuskens I, Michalik M, Chauhan N, Linke D, Leo JC. A new strain collection for improved expression of outer membrane proteins. Front Cell Infect Microbiol. 2017;7:464. https://doi.org/10.3389/fcimb.2017.00464.

30. Meuskens I, Saragliadis A, Leo JC, Linke D. Type V secretion systems: an overview of passenger domain functions. Front Microbiol. 2019;10:1163.

31. Nicolay T, Vanderleyden J, Spaepen S. Autotransporter-based cell surface display in Gram-negative bacteria. Crit Rev Microbiol. 2015;41:109-23.

32. Noinaj N, Gumbart JC, Buchanan SK. The $\beta$-barrel assembly machinery in motion. Nat Rev Microbiol. 2017;15:197-204.

33. Otto BR, Sijbrandi R, Luirink J, Oudega B, Heddle JG, Mizutani K, Park SY, Tame JR. Crystal structure of hemoglobin protease, a heme binding autotransporter protein from pathogenic Escherichia coli. J Biol Chem. 2005;280:17339-45

34. Prilipov A, Phale PS, Van Gelder P, Rosenbusch JP, Koebnik R. Coupling site-directed mutagenesis with high-level expression: large scale production of mutant porins from E. coli. FEMS Microbiol Lett. 1998;163(1):65-72. https://doi.org/10.1111/j.1574-6968.1998.tb13027.x.

35. Ramesh B, Sendra VG, Cirino PC, Varadarajan N. Single-cell characterization of autotransporter-mediated Escherichia coli surface display of disulfide bond-containing proteins. J Biol Chem. 2012;287:38580-9.

36. Reed B, Chen R. Biotechnological applications of bacterial protein secretion: from therapeutics to biofuel production. Res Microbiol. 2013;164:675-82

37. Roman-Hernandez G, Peterson JH, Bernstein HD. Reconstitution of bacterial autotransporter assembly using purified components. Elife. 2014;3:e04234.

38. Salema V, Marín E, Martínez-Arteaga R, Ruano-Gallego D, Fraile S, Margolles Y, Teira X, Gutierrez C, Bodelón G, Fernández LÁ. Selection of single domain antibodies from immune libraries displayed on the surface of E. coli cells with two $\beta$-domains of opposite topologies. PLOS ONE. 2013;8:e75126.

39. Sauri A, Oreshkova N, Soprova Z, Jong WS, Sani M, Peters PJ, Luirink J, van Ulsen P. Autotransporter beta-domains have a specific function in protein secretion beyond outer-membrane targeting. J Mol Biol. 2011:412:553-67.

40. Sauri A, Soprova Z, Wickstrom D, de Gier JW, Van der Schors RC, Smit AB, Jong WSP, Luirink J. The Bam (Omp85) complex is involved in secretion of the autotransporter haemoglobin protease. Microbiology (Reading). 2009;155:3982-91.

41. Sauri A, Ten Hagen-Jongman CM, van Ulsen P, Luirink J. Estimating the size of the active translocation pore of an autotransporter. J Mol Biol. 2012:416:335-45.

42. Schetters STT, Jong WSP, Horrevorts SK, Kruijssen LJW, Engels S, Stolk D, Daleke-Schermerhorn MH, Garcia-Vallejo J, Houben D, Unger WWJ, den Haan JMM, Luirink J, van Kooyk Y. Outer membrane vesicles engineered to express membrane-bound antigen program dendritic cells for crosspresentation to CD8(+) T cells. Acta Biomater. 2019;91:248-57.

43. Schlapschy M, Grimm S, Skerra A. A system for concomitant overexpression of four periplasmic folding catalysts to improve secretory protein production in Escherichia coli. Protein Eng Des Sel. 2006;19:385-90.

44. Schlapschy M, Skerra A. Periplasmic chaperones used to enhance functional secretion of proteins in E. coli. Methods Mol Biol. 2011;705:211-24.

45. Sikdar R, Bernstein HD. Sequential translocation of polypeptides across the bacterial outer membrane through the trimeric autotransporter pathway. MBio. 2019;10:e01973-19. 
46. Sikdar R, Peterson JH, Anderson DE, Bernstein HD. Folding of a bacterial integral outer membrane protein is initiated in the periplasm. Nat Commun. 2017:8:1309

47. Soprova Z, Sauri A, van Ulsen P, Tame JR, den Blaauwen T, Jong WS, Luirink J. A conserved aromatic residue in the autochaperone domain of the autotransporter $\mathrm{Hbp}$ is critical for initiation of outer membrane translocation. J Biol Chem. 2010;285:38224-33.

48. Steenhuis M, Abdallah AM, de Munnik SM, Kuhne S, Sterk GJ, van den Berg van Saparoea B, Westerhausen S, Wagner S, van der Wel NN, Wijt-

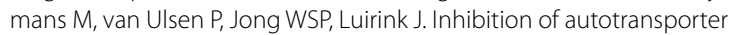
biogenesis by small molecules. Mol Microbiol. 2019;112:81-98.

49. Tomasek D, Rawson S, Lee J, Wzorek JS, Harrison SC, Li Z, Kahne D. Structure of a nascent membrane protein as it folds on the BAM complex. Nature. 2020;583(7816):473-8. https://doi.org/10.1038/ s41586-020-2370-1.

50. Tozakidis IE, Brossette T, Lenz F, Maas RM, Jose J. Proof of concept for the simplified breakdown of cellulose by combining Pseudomonas putida strains with surface displayed thermophilic endocellulase, exocellulase and $\beta$-glucosidase. Microb Cell Fact. 2016;15:103.

51. Valle J, Mabbett AN, Ulett GC, Toledo-Arana A, Wecker K, Totsika M, Schembri MA, Ghigo JM, Beloin C. UpaG, a new member of the trimeric autotransporter family of adhesins in uropathogenic Escherichia coli. J Bacteriol. 2008;190:4147-61.

52. van den Berg van Saparoea HB, Houben D, de Jonge MI, Jong WSP, Luirink J. Display of recombinant proteins on bacterial outer membrane vesicles by using protein ligation. Appl Environ Microbiol. 2018;84:e02567-17.
53. van Dooren SJ, Tame JR, Luirink J, Oudega B, Otto BR. Purification of the autotransporter protein $\mathrm{Hbp}$ of Escherichia coli. FEMS Microbiol Lett. 2001;205:147-50.

54. van Ulsen P, Zinner KM, Jong WSP, Luirink J. On display: autotransporter secretion and application. FEMS Microbiol Lett. 2018;365:fny165.

55. Wagner S, Klepsch MM, Schlegel S, Appel A, Draheim R, Tarry M, Högbom M, van Wijk KJ, Slotboom DJ, Persson JO, de Gier JW. Tuning Escherichia coli for membrane protein overexpression. Proc Natl Acad Sci USA. 2008;105:14371-6.

56. Webb CT, Heinz E, Lithgow T. Evolution of the $\beta$-barrel assembly machinery. Trends Microbiol. 2012;20(12):612-20. https://doi.org/10.1016/j.tim. 2012.08.006.

57. Zakeri B, Fierer JO, Celik E, Chittock EC, Schwarz-Linek U, Moy VT, Howarth M. Peptide tag forming a rapid covalent bond to a protein, through engineering a bacterial adhesin. Proc Natl Acad Sci USA. 2012;109:E690-7.

58. Zhang M, Tanaka T, Ikura M. Calcium-induced conformational transition revealed by the solution structure of apo calmodulin. Nat Struct Biol. $1995 ; 2: 758-67$

\section{Publisher's Note}

Springer Nature remains neutral with regard to jurisdictional claims in published maps and institutional affiliations.
Ready to submit your research? Choose BMC and benefit from:

- fast, convenient online submission

- thorough peer review by experienced researchers in your field

- rapid publication on acceptance

- support for research data, including large and complex data types

- gold Open Access which fosters wider collaboration and increased citations

- maximum visibility for your research: over $100 \mathrm{M}$ website views per year

At BMC, research is always in progress.

Learn more biomedcentral.com/submissions 